\title{
Editorial
}

\section{A revolução dos dados e a nova ciência das cidades}

\author{
Vinicius M. Netto \\ Julio Celso Vargas \\ Renato T. de Saboya \\ Editores da Revista de Morfologia Urbana
}

https://doi.org/10.47235/rmu.v8i1.173

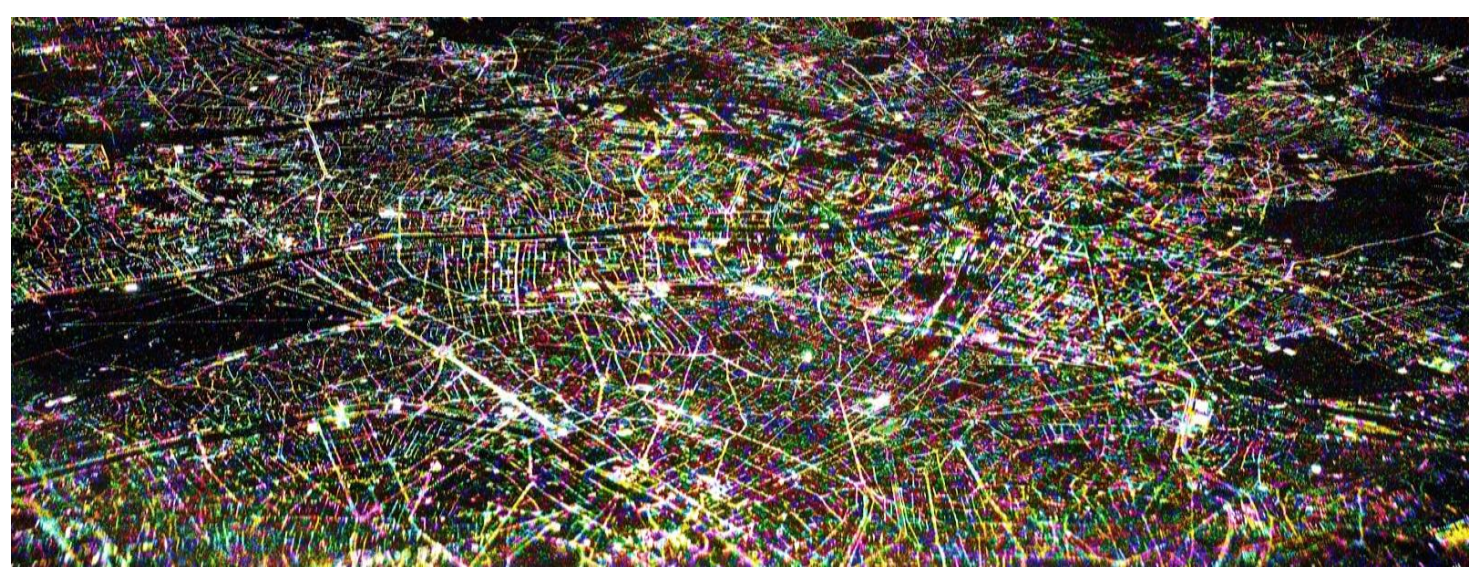

Figura 1. Lugares e ações urbanas representados em fluxos dinâmicos de dados (fonte: adaptada por Vinicius Aleixo sobre foto de Dennis Kummer, disponível em https://unsplash.com/photos/52gEprMkp7M).

Um intenso movimento tem agitado os estudos urbanos e suas fronteiras. Esse movimento é disparado por nossas ações na própria cidade, registradas e reverberadas por smartphones e gadgets que carregamos conosco o tempo todo, por nossos computadores e pela Internet, assim como por sensores e dispositivos inseridos no próprio tecido da cidade. O movimento é feito de dados — os fluxos invisíveis e aparentemente onipresentes de bits, os registros eletrônicos de nossos comportamentos, da cidade e de seu funcionamento. Muitos termos vêm sendo usados para descrevê-lo: onda, tsunami, oceano. Essas metáforas são sugestivas, mas ainda dizem pouco do que acontece e do que está por vir.

Os estudos urbanos e o campo da morfologia urbana em particular estão mudando. Como pesquisadores imersos no túnel dos nossos sentidos e experiências, estávamos até recentemente limitados por tecnologias capazes de registrar apenas seções e partes do ambiente a nossa volta (Netto, 2014). A leitura alocêntrica de uma cidade e de seu funcionamento como um todo em sua infinidade de fluxos e eventos simultâneos, muitos deles conectados, evadia nossos recursos cognitivos e técnicos. $\mathrm{O}$ entendimento da cidade como um todo sempre demandou a imaginação — auxiliada por teorias e registros, métodos e modelos. Esses recursos de alguma forma nos aproximaram do fenômeno complexo que é a cidade - uma totalidade formada pelas ações e interações em números e possibilidades astronômicas entre pessoas e entre lugares, produzindo tramas de movimentos de diferentes ritmos e materialidades: corpos, objetos, veículos, informação. Mas enquanto sistema de sistemas — humanos e técnicos, físicos e cognitivos sobrepostos, convergentes, alguns deles apenas parcialmente visíveis - a cidade provou ser algo que escapava a nossos meios para conhecê-la. A cidade sempre evadiu nosso entendimento.

É essa limitação que os dispositivos eletrônicos que trazemos junto a nossos corpos, nossos computadores e os sensores incorporados na própria estrutura da cidade 
— em edifícios, lugares públicos, veículos vêm permitindo reduzir. Extraindo e registrando a cada segundo fluxos de dados ao nível das ações e rotinas pessoais esses dispositivos oferecem um raio- $x$ sem precedentes de nossas cidades, incluindo novas possibilidades de entender suas dinâmicas em tempo real. Eles permitem uma leitura ampla e sistêmica, ao mesmo tempo que rica e detalhada de cidades e de seus tantos processos. Check-ins em lugares da cidade são voluntariamente lançados nas mídias sociais digitais. Movimentos individuais são constantemente capturados por telefones celulares. Dados associados a cartões de usuários de sistemas de transporte público ou gerados por sensores instalados em veículos possibilitam penetrar nas diferentes camadas da mobilidade urbana e visualizar os fluxos e ritmos extraordinários da cidade. Esses dados possibilitam observar comportamentos espaciais na escala do indivíduo e conhecer como eles se somam em direções e padrões coletivos. Algoritmos ainda vêm permitindo a pesquisadores 'customizar' suas análises em níveis de desagregação sem precedência. Em outras frentes de pesquisa, estudos empíricos têm abordado centenas e mesmo milhares de cidades em diferentes regiões do mundo, estabelecendo novos patamares de robustez empírica para a disciplina.

Esses recursos têm permitido visualizar as conexões entre enormes quantidades de pessoas em redes sociais presenciais amarradas pelos nós que são os lugares urbanos - redes antes impossíveis de reconstruir. Têm permitido acompanhar o movimento e as flutuações de mercados sob os impactos de plataformas digitais da economia compartilhada. Possibilitam monitorar a emissão de poluentes em nossos espaços públicos e confrontá-los com dados da saúde de populações. Esses novos recursos têm alimentado o interesse em cidades como sistemas complexos (veja Krafta e Rauber, nesta edição), assim como as aproximações com a Física, uma disciplina na qual lidar com um número enorme de entidades relacionadas de modo não necessariamente linear, com precisão no uso de dados, é procedimento comum (veja Ribeiro, nesta edição).

Esses são movimentos disparados por contatos, convergências e mesmo confrontos entre ciência e tecnologia. A explosão de informação (data deluge), os novos recursos técnicos e a disseminação da capacidade de processar dados em grandes volumes vêm alimentando métodos de pesquisa antigos e novos. Vemos novas pontes sendo rapidamente construídas entre diferentes áreas do conhecimento, incluindo os estudos urbanos, e a emergente ciência de dados (data science), cruzando computação, matemática e estatística - um 'quarto paradigma' científico depois do empírico, teórico e computacional, orientado por dados (data-driven - veja Hey, Tansley e Tolle, 2016).

A sensação pode ser de vertigem, mas esses movimentos e contatos não são livres de contradições e riscos. Ainda que muitas inovações tenham sido inicialmente alimentadas por recursos públicos em universidades e agências governamentais, como a máquina de busca do Google, smartphones e a própria fundação da Internet (Singer, 2014), as tecnologias de dados e suas aplicações são com frequência desenvolvidas e disponibilizadas por corporações de diferentes lugares do mundo, mas sobretudo localizadas na Califórnia, Estados Unidos. Considerando sua origem e a concentração de inovações em um pequeno grupo de imensas empresas, a produção e uso de tecnologia vem tendo um papel central na ampliação das desigualdades entre países e no chamado 'fosso digital' (digital divide) entre indivíduos (Rodriguez e Wilson, 2000; Brian, 2015). Uma ciência que clama buscar cidades mais eficientes e igualitárias tem suas ferramentas desenvolvidas em países ricos, ignorando as dificuldades de aplicação aos problemas dos países em desenvolvimento, atravessados por escassez de recursos (veja Gamboa e Demerutis, nesta edição) e por desigualdades internas de acesso à informação (veja Feitosa, nesta edição). Há ainda riscos severos das tecnologias de registros digitais afetarem a privacidade de populações, com a exploração comercial de dados pessoais e a possibilidade do escrutínio de comportamentos como nunca antes.

Recursos de mapeamento facial capazes de impor o fim do anonimato no espaço público ilustram a seriedade do problema. A possibilidade de controle dos dados permite ainda tentativas de ocultação e invisibilização de dados públicos (veja Firmino et al., nesta edição) e de usos para fins de controle social, potencializando formas de totalitarismo. 
A onda traz consigo uma infinidade de termos novos, frequentemente sem tradução: big data, data analytics, smart cities, machine learning, inteligência artificial. Esses termos ganham rápida popularidade, tomando os vocabulários de outras regiões do mundo, e denotam coisas com relevâncias variadas da revolução digital. Um desses termos é o digital twin (gêmeo digital). Trata-se da criação da imagem espelhada de um processo físico - como a representação das edificações de uma cidade e sua operação ou consumo de energia, ou como a representação computacional dos fluxos veiculares em uma cidade. $O$ gêmeo digital é comparado em tempo real com o processo em questão, replicando sua dinâmica e funcionamento. $\mathrm{O}$ termo caracteriza ainda uma variedade de modelos de simulação que rodam paralelamente a processos urbanos, sociais ou econômicos em andamento, recebendo input dos sistemas reais. A ideia foi introduzida por Michael Grieves no começo dos anos 2000, mas só recentemente ganhou popularidade. Naturalmente, $o$ desafio é definir as diferenças entre o sistema real e o modelo computacional daquele sistema (Batty, 2018). Esses modelos digitais podem ainda receber informação em tempo real do funcionamento dos seus gêmeos 'concretos' - ecoando a ideia da 'internet das coisas', segundo a qual dispositivos e máquinas se comunicam e coordenam seu funcionamento, acessando-se mutuamente.

\section{As implicações para a pesquisa e o} planejamento dos modelos digitais poderão ser muitas. Em tese, esses modelos podem permitir testar os limites do conhecimento no confronto com o processo ou sistema urbano. Se acurados, os gêmeos digitais permitirão verificar alterações e propostas de planejamento antes de aplicá-las no contexto real, possibilitando entender efeitos e cenários via resultados e visualizações capazes de informar intervenções próximas ao tempo real, em princípio podendo auxiliar o funcionamento de uma cidade (Batty, 2018). Se esses modelos ainda não rodam em tempo real acompanhando seus gêmeos concretos, esse é o horizonte buscado. A ideia está conectada ainda ao conceito de smart cities - quando sistemas digitais não são apenas 'gêmeos' mas ligados aos sistemas urbanos, alimentando-os como instrumentos de coordenação de operações, ligando modelos ao ambiente construído e ligando o funcionamento a processos físicos e dinâmicas socioeconômicas - por exemplo, o uso de softwares capazes de indicar a demanda por tipos de transporte e guiar a oferta de ônibus ou trens a seus usuários.

Essencialmente, smart cities são cidades nas quais novas tecnologias digitais de informação e comunicação são integradas à infraestrutura, capazes de monitorar o funcionamento de subsistemas e dinâmicas urbanas, e passam a reger a coordenação desses subsistemas e processos. Essas tecnologias incluem, além de recursos computacionais, dispositivos de sensoriamento acoplados a estruturas físicas, de edifícios a sistemas de transporte, capazes de gerar dados sobre o funcionamento urbano em diferentes escalas de espaço e tempo. Fazem uso de recursos de simulação urbana capazes de identificar riscos, incertezas e problemas críticos urbanos associados ao transporte, energia e ambiente, e capazes de informar decisões futuras. A ideia de smart cities ainda sugere a possibilidade de integração com tecnologias de comunicação e disseminação capazes de alimentar novas formas de governança urbana e organização social. Inclui relacionar a infraestrutura da cidade e seu funcionamento ao planejamento e gestão, com o objetivo de otimizar recursos. Em princípio, esses meios podem ser usados para dar suporte ao acesso a oportunidades urbanas de acordo com valores de governança democrática, estimulando a equidade e justiça (Batty et al., 2012).

Entretanto, o conceito de smart cities vem antes sendo apropriado por empresas e comercializado na forma de serviços e marketing (veja Gamboa e Demerutis, nesta edição).

Parece haver um longo caminho para que o potencial democrático da revolução de dados seja de fato realizado. Uma possibilidade é a convergência dessas tecnologias de informação e coordenação em aplicações para além da esfera governamental ou institucional. Há um potencial político que não deve ser ignorado, sobretudo se essas tecnologias forem hackeadas em ações coletivas 'de baixo para cima' (bottom-up). Elas podem empoderar agências sociais via plataformas digitais participativas de captura e troca de dados e informação (sensing platforms) - por exemplo, para a coleta de percepções de cidadãos e stakeholders em relação a seus lugares, e 
sobre as dificuldades que enfrentam ao navegar pela cidade. Em outras palavras, tecnologias de captura de dados em convergência com a Internet permitem gerar plataformas de troca de conhecimento, coletando informações em diferentes áreas das cidades, permitindo que moradores de diferentes gêneros, idades e origens étnicas avaliem seus bairros e compartilhem suas leituras. Trata-se da possibilidade de conexão direta entre atores urbanos, um modo de informar e aprender coletivamente a partir de experiências individuais. Integrando desde percepções subjetivas até entendimento sistêmico, essas plataformas devem permitir identificar problemas de mobilidade, desempenho e justiça na distribuição espacial de oportunidades na microescala. São ainda uma forma de acessar dados altamente desagregados com potencial tanto para a pesquisa quanto para o planejamento, permitindo comparações com análises técnicas e cenários simulados. Veremos nesta edição de RMU exemplos de como essas plataformas podem emergir de ações individuais e se tornar espaços de voz para grupos sociais à margem das políticas oficiais no contexto brasileiro - na Perspetiva de Rodrigo Firmino, Debora Pio e Gilberto Vieira.

Finalmente, a revolução dos dados alimenta a chamada 'nova ciência das cidades'. A publicação do livro The New Science of Cities por Mike Batty em 2013 já anunciava mudanças radicais na noção do que a cidade é. Uma dessas mudanças é a passagem da ideia de sistema cujo funcionamento buscava equilíbrio associado à organização top-down, de cima para baixo, típica das abordagens funcionalistas, ao reconhecimento da complexidade de comportamentos subjacentes e na ideia de 'emergência' de dinâmicas urbanas a partir de decisões e ações de baixo para cima, que podem por sua vez levar a comportamentos coletivos inesperados (Batty, 2012). Temos a passagem da análise de fenômenos com números finitos ou reconhecíveis de componentes para a análise de fenômenos compostos por um número imensurável ou desconhecido de variáveis interconectadas de forma potencialmente não-linear e atravessados por contingências, como é o caso das cidades (veja Ribeiro, nesta edição). Temos ainda a mudança do foco na localização, tradicional na geografia econômica e estudos urbanos, ao foco nas interações como a chave para entender cidades como redes de diferentes materialidades sobrepostas e conectadas (Batty, 2013), e como força motriz da construção de redes sociais - a cidade como hardware, software e 'reator' de interações (Bettencourt, 2013; cf. Jacobs, 1969). Como Meirelles e colegas afirmam em seu artigo nesta edição, as abordagens da NCC ainda se concentram não nas particularidades das cidades, mas em seus padrões comuns.

Pesquisas vêm explorando bancos de dados de centenas a milhares de cidades e descobrem padrões indutivamente, como a já bem conhecida 'lei de escala' ditando comportamentos de aspectos urbanos na medida em que cidades assumem portes diferentes. Evidências empíricas mostram que o tamanho da população está fortemente relacionado com aspectos de comportamento urbano. Variáveis socioeconômicas como o PIB e renda escalam de forma superlinear quando cidades aumentam o tamanho da sua população - ou seja, escalam mais do que proporcionalmente. Por exemplo, se uma cidade tem o dobro da população que outra (100\% mais população), seu PIB, renda per capita e o número de patentes registradas tendem a ser em torno de $115 \%$ superiores (Bettencourt, 2013; Meirelles et al, 2018 e nesta edição). Pessoas falam mais ao celular e caminham mais rápido, mostrando que nossas interações se intensificam em cidades maiores (Schläpfer et al, 2014). Outros fatores urbanos, como estrutura e infraestrutura urbana, escalam de forma sublinear (menos que proporcionalmente) com o número de habitantes. Isso quer dizer que, quando o tamanho da cidade aumenta, o número per capita destas quantidades, como a extensão das redes de ruas, tende a diminuir (Ribeiro et al., 2020).

Os dois processos ocorrem por causa das extraordinárias propriedades das redes que estruturam as cidades e conectam lugares, nas quais nossos corpos, objetos e informação se movimentam e ganham eficiências quando adicionamos mais pessoas, edificações e lugares a elas, maximizando o uso das estruturas e infraestruturas físicas e intensificando o contato e a conectividade entre as pessoas (Netto et al., 2020). Essas possibilidades de investigação impulsionam uma ciência urbana agora livre para a pesquisa indutiva — olhando primeiro para dados volumosos, 
tentando capturar o que emerge da aparente aleatoriedade na forma de padrões reconhecíveis. As novas tecnologias de dados trazem ainda controvérsias sobre $o$ fenômeno urbano e sua universalidade, junto a riscos de um neopositivismo, do determinismo tecnológico e da redução do urbano a um universo de sistemas ao largo dos mundos subjetivos de seus atores.

Esses e outros tantos aspectos de uma nova ciência urbana ancorada na chamada revolução dos dados digitais clamam por escrutínio e debate - nossos objetivos nesta publicação coletiva.

\section{A chamada de artigos}

Ao trazer como tema "A nova ciência das cidades e a revolução dos dados", a Revista de Morfologia Urbana busca oferecer um conjunto de trabalhos capazes de introduzir pesquisadores lusófonos nesse universo. $\mathrm{O}$ objetivo é reunir leituras do que esses movimentos são e de seus impactos tanto na pesquisa em estudos urbanos e no campo da morfologia urbana em particular quanto nas práticas urbanas, no planejamento e no próprio futuro das cidades - como discutem Romulo Krafta e Alice Rauber em seu artigo convidado. Nessa espécie de guia aberto construído coletivamente nestas páginas, veremos discussões, leituras e aplicações que nos remetem a questões como:

- O que é a nova ciência das cidades $(N C C)$ ? Por que ela seria uma nova ciência? Entendendo 'ciência' como uma forma rigorosa de relacionar proposições teóricas a recursos metodológicos voltados para compreender problemas empíricos e permitir o suporte (ou rejeição) daquelas proposições iniciais, por que essa é uma nova ciência? Seria uma única ciência, ou seriam muitas as ciências da cidade? Qual é a diferença entre ela e a ciência anterior das cidades, se é que ela existiu?

- Centros de pesquisa urbana no mundo todo vêm ganhando o termo 'ciência urbana' (ou variações dele) em seus nomes. Eventos acadêmicos carregam o termo como uma marca. Por que o termo vem ganhando tanta atenção?

- A nova ciência das cidades parece ter um paralelo anterior na revolução quantitativa na geografia na década de 1960. Aquele movimento, alimentado pela introdução de computadores (ainda de grande porte e uso restrito a centros de pesquisa) logo encontrou forte ceticismo e resistência, desencadeando um novo movimento conhecido como 'geografia crítica' e uma ênfase em abordagens interpretativas. O que há de diferente na revolução de dados? É uma mera consequência da tecnologia e do poder crescente dos computadores, que acabará sendo rejeitada por visões críticas?

- O que a crescente ênfase tecnológica e uso de dados quantitativos significam para as abordagens interpretativas e qualitativas, e para pesquisadores interessados nessas outras formas de pesquisa? Como evitar o aprofundamento de um dualismo quantitativo versus qualitativo?

- O que a revolução dos dados representa para os estudos urbanos e para a morfologia urbana em particular? Seria uma nova forma de reconhecer e entender o urbano - uma forma incontornável, pronta para engolir a disciplina e o campo, e desafiá-los em seus fundamentos e bases empíricas?

- Como a nova ciência das cidades atinge a pesquisa lusófona, assim como a pesquisa no sul global e nos países em desenvolvimento, como na América Latina e África?

- As cidades parecem ter características em comum o suficiente para permitir que sejam chamadas de 'cidades', um mesmo fenômeno que se manifesta em infinitas formas. Internamente, elas contêm relações fortes o bastante para permitir descobertas como a Lei de Zipf em distribuições de tamanho e escala. Quais são esses elementos urbanos em comum e como a NCC é capaz de revelá-los? Por outro lado, cada cidade é um fenômeno próprio, com características particulares fortes o suficiente para lhes dar identidades. Como a nova ciência das cidades lida com essas identidades e idiossincrasias? Como ela pode capturar a 'alma' de uma cidade - aquilo que a torna única?

Essas são questões difíceis, cujas respostas estão ainda em construção. Elas provavelmente serão trazidas, avaliadas e 
reformuladas em diferentes interpretações e correntes.

A RMU convocou artigos científicos e a leitura de especialistas que exploram esses e outros aspectos, sob a forma de (a) trabalhos empíricos no espírito da NCC e do uso rigoroso de dados, e (b) discussões teóricas, conceituais e/ou críticas da NCC, da chamada 'revolução dos dados' e das aplicações de tecnologias da informação no acompanhamento de dinâmicas urbanas e sociais. Dada a grande quantidade de submissões, esta chamada se desdobrará nas duas edições de 2020. Vejamos agora o que este primeiro número traz.

\section{A presente edição: artigos e perspetivas}

Nesta edição temática da Revista de Morfologia Urbana, temos as contribuições de uma série de pesquisadores compondo a seção de artigos científicos e a seção "Perspetivas".

Convidamos Romulo Krafta e Alice Rauber para abrir a seção temática de artigos científicos, com sua ampla leitura "Morfologia urbana e a revolução dos dados". Krafta é provavelmente o pesquisador que mais contribuiu para introduzir o que chamava 'ciência urbana' como abordagem à cidade como sistema complexo no contexto brasileiro. Rompendo resistências e desenvolvendo teoria sobretudo desde os anos 1990, vem formando e influenciando novas gerações de pesquisadores, ajudando a estabelecer uma cultura de pensamento configuracional no mundo lusófono. Em seu artigo, Krafta e Rauber oferecem um panorama das formas e ganhos da revolução de dados no campo da morfologia urbana, numa descrição de sua transformação - desde as dificuldades de tratamento dos artefatos urbanos em função de "enormes quantidades de componentes, derivando quantidades inimagináveis de possíveis configurações" (p.12). Entram ainda em território prospectivo, explorando e imaginando expansões da abordagem topológica em direção a diferentes camadas materiais, cognitivas e temporais como caminhos para a "explanação definitiva do fenômeno urbano" (p.10).

$\mathrm{O}$ artigo "Evolução das leis de escala urbanas: evidências do Brasil", de João Meirelles, Camilo Rodrigues Neto, Fernando Ferreira, Fabiano Ribeiro e Claudia Binder, traduzido por Julio Celso Vargas, examina o comportamento de municípios brasileiros sob a luz da mencionada 'lei de escala urbana'. Os autores examinam se as mais de 60 variáveis socioeconômicas, de infraestrutura e de serviços levantadas nesses municípios variam em função do tamanho de suas populações, a partir de um certo limiar de densidade. Em outras palavras, investigam se a suposta universalidade do princípio do escalamento ora superlinear, linear ou sublinear é válido para cidades de países com diferentes estágios de desenvolvimento. Finalmente, detectam idiossincrasias no contexto brasileiro que podem ainda ser explicadas pela influência de ações de planejamento top-down, sobretudo em fatores de infraestrutura.

Em "Identificação de composições da paisagem urbana: uma abordagem de deep learning", Ana Luiza Favarão Leão, Hugo Queiroz Abonizio, Sylvio Barbon Júnior e Milena Kanashiro ilustram o potencial dos recursos digitais para abordar a estética, qualidades espaciais e a composição da paisagem urbana. $\mathrm{O}$ artigo explora procedimentos de análise baseados em técnicas de machine learning ('aprendizado de máquina') e deep learning, com suas muitas camadas de processamento e transformação de representações em redes neurais para identificar elementos compositivos da paisagem e gerar classificações de características espaciais e paisagísticas e associá-las a bairros de origem, a partir de um banco de imagens do Google Street View. Usando mais de 2000 imagens colhidas em bairros selecionados em Londrina, Paraná, sul do Brasil, a abordagem mostra o potencial do 'aprendizado de máquina' para lidar com grandes amostras por via de recursos empíricos digitais na detecção de padrões formais no ambiente construído.

Karine de Almeida Paula, Eleusy Miguel e Wagner Freitas investigam em "A cidade patrimonial e turística: uma análise do constructo da imagem e apropriação do centro histórico de Tiradentes $-M G$ a partir das mídias sociais" a percepção de turistas em um contexto urbano com características patrimoniais a partir dos dados publicados em mídias digitais de registros fotográficos e a técnica de mineração de dados Web Scraping (WS). A exploração desse método permitiu aos autores identificar a imagem 
urbana formada a partir dessas percepções e registros, apontando sua associação ao Centro Histórico e a relativa redução da cidade ao espaço central de Tiradentes.

A seção Perspetivas traz textos de autores convidados. Fabiano L. Ribeiro nos mostra em "A Física das Cidades" o potencial da aproximação entre sua disciplina de origem e os estudos urbanos, uma das fronteiras da nova ciência das cidades. Na busca pelo entendimento da natureza das cidades, Ribeiro, como Mike Batty, nos lembra da importância da teoria para penetrar no imenso volume de dados gerados a cada segundo pelos numerosos agentes vivendo a cidade e para produzir conhecimento científico. Discutindo as leis de escala urbana e as propriedades fractais da cidade, usa como metáforas os diferentes estágios da Física: a fase 'Brahe', focada na obtenção de dados de um fenômeno; a fase 'Kepler', que penetra os dados para reconhecer certos padrões, e a fase 'Newton', em que as informações anteriores geram explicações do fenômeno. Ribeiro ainda discute a diferença entre uma ciência das cidades e uma ciência para as cidades.

Uma perspectiva latino-americana da nova ciência urbana e a ênfase em dados é trazida por Mayra Eugenia Gamboa González e Juan Ángel Demerutis Arenas, professores da Universidad de Guadalajara, no México, em "Big Data and Urban Analytics: City Science in Developing Economies". Situando a tecnologia como extensão da cognição humana, apontam os abusos do uso do termo 'smart city' e afirmam a interdisciplinaridade da ciência urbana, ao reconhecer cidades como sistemas de sistemas - social, político, ecológico-territorial. Os autores discutem ainda o status de uma nova disciplina que clama perseguir cidades mais eficientes e igualitárias, mas tem suas ferramentas criadas sobretudo em países desenvolvidos. Trazem questões sobre como essa ciência pode ser aplicada para os problemas em sociedades em desenvolvimento, imersos em desigualdades, recursos limitados e divisão digital, incluindo a dificuldade de grupos sociais vulneráveis em absorver as novas tecnologias. Analisando a atividade de novos centros de pesquisa em ciência urbana, Gamboa e Demerutis levantam a possibilidade da produção e uso de tecnologia intensificar desigualdades sociais, e tecem uma leitura da aplicações concretas da ciência urbana em problemas urbanos e a qualidade de vida de comunidades.

Finalmente, discutem a possibilidade de implementação de uma ciência urbana mais afeita às necessidades das cidades na América Latina, considerando seus desafios institucionais e materiais e a necessidade de abordagens que tenham regiões específicas como referencial.

Em "Big Data e Urban Analytics à brasileira: questões inerentes a um país profundamente desigual', Flávia Feitosa recomenda cuidados com o entusiasmo com a emergente ciência de dados e a chamada urban analytics, enfatizando o papel dos dados tradicionais e a possibilidade de integração ao big data para potencializar o uso de ambos. Situa a revolução dos dados na realidade urbana brasileira, discutindo limitações de capacidade de armazenamento e tratamento sistemático de dados, abrangência geográfica fragmentada e desigualdade de informação entre e dentro de cidades - e seus impactos no potencial dos dados digitais para avanços na pesquisa e planejamento, para a compreensão da natureza do urbano, a necessidade de uma política de abertura dos dados - a conversão do big data em open data - e a possibilidade da descentralização do 'pensar a cidade' em contextos de invisibilidade social.

Rodrigo Firmino, Debora Pio e Gilberto Vieira trazem em "Revolução periférica dos dados em tempos de pandemia global" a visão de como as novas tecnologias e plataformas digitais de informação e comunicação vêm sendo apropriadas em contra-culturas e por grupos sociais nãohegemônicos - conectando a edição temática com a urgência da crise de saúde pública que vivemos. Os autores descrevem a coleta e manipulação de dados como ações políticas, parte da definição de políticas públicas e fundamentais para a visibilidade de grupos sociais e territórios vulnerabilizados. Descrevem a organização de coletivos em favelas e periferias urbanas em redes de resistência e arranjos sociotécnicos em resposta à pandemia, e em resposta à sua invisibilidade aos órgãos oficiais de informação urbana e de saúde pública. A reflexão penetra nas redes organizadas a partir de mídias sociais e do acesso a telefones celulares conectados à internet - muitas vezes 'hackeando' a 
cidade e suas infraestruturas formais em táticas de um 'ativismo de sobrevivência' incluindo orientação, ajuda mútua e financiamento coletivo de mantimentos e contato com autoridades públicas, além do uso de recursos de espacialização da informação como mapas colaborativos - um novo tipo de resistência e ação política na margem, via tecnologias e dados digitais.

A seção aberta de RMU traz ainda dois artigos. Milton Conceição investiga em "Questões fronteiriças: Florianópolis e Olivença, duas faces da mesma moeda" os papéis das cidades de Olivenza (Espanha) e Florianópolis (Brasil) na disputa entre lusos e castelhanos nas fronteiras ibéricas e na costa atlântica sul das Américas, e a materialidade engendrada por eventos históricos.

Em "Avaliação de vizinhança LEED $e$ análise microclimática: um estudo de caso

\section{Referências}

Batty, M. (2018) Digital twins. Environment and Planning B: Urban Analytics and City Science, 45(5), 817-820. Disponível em: https://doi.org/10.1177/2399808318796416.

Batty, M. (2013) The New Science of Cities. Cambridge, The MIT Press.

Batty, M. (2012) Building a science of cities. Cities, 29, S9-S16.

Batty, M.; Axhausen, K. W.; Giannotti, F.; Pozdnoukhov, A.; Bazzani, A.; Wachowicz, M. e Portugali, Y. (2012) Smart cities of the future. The European Physical Journal Special Topics, 214(1), 481-518.

Bettencourt, L. M. A. (2013) The origins of scaling in cities. Science, 340, 1348-1441.

Brian, K. (2015) OECD insights income inequality the gap between rich and poor. Organisation for Economic Co-operation and Development. OECD Insights, OECD Publishing, Paris. Disponível em: http://dx.doi.org/10.1787/9789264246010-en.

Jacobs, J. (1969) The Economy of Cities. New York, Random House.

Meirelles, J.; Rodrigues Neto, C.; Ferreira, F.F.; Ribeiro, F. L. e Binder, C. R. (2018) Evolution of urban scaling: evidence from Brazil. Plos One, 10, 1-15.

Netto, V. M. (2014) Cidade \& sociedade: as tramas da prática e seus espaços. Capítulo 5 "Entre espaços urbanos e digitais". Porto Alegre, Editora Sulina. da morfologia urbana do Porto Maravilha, RJ, Brasil', Amanda Marques da Silva, Gisele Barbosa e Patricia Drach exploram simulações computacionais para analisar possíveis efeitos climáticos disparados por mudanças de legislação urbana e consequentes alterações morfológicas trazidas por um grande projeto de revitalização. Esses cenários são então comparados a situações hipotéticas baseadas nos requisitos do sistema LEED-

Neighborhood e em critérios de urbanismo sustentável.

Finalmente, esta edição celebra o talento, a criatividade e a vida do inesquecível João Vitor Meirelles - jovem pesquisador, coautor e querido amigo.

Netto, V. M.; Ribeiro, F. L., Carvalho, C.L.S.; Cabral, V. e Neto, C.R. (2020) As cidades na pandemia: o papel do tamanho e da densidade urbana. Caos Planejado. Disponível em: https://caosplanejado.com/as-cidades-napandemia-o-papel-do-tamanho-e-dadensidade-urbana/. [Consultado em: 16 de junho de 2020].

Ribeiro, F. L., Meirelles, J., Netto, V. M., Neto, C. R., e Baronchelli, A. (2020). On the relation between Transversal and Longitudinal Scaling in Cities. arXiv preprint arXiv:1910.02113.

Ribeiro, F. L., Meirelles, J., Netto, V. M., Neto, C. R., e Baronchelli, A. (2020). On the relation between Transversal and Longitudinal Scaling in Cities. arXiv preprint arXiv:1910.02113.

Singer, P.L. (2014) Federally supported innovations: 22 examples of major technology advances that stem from federal research support. Report for The Information Technology \& Innovation Foundation. Washington, DC. Disponível em: http://www2.itif.org/2014-federally-

supported-innovations.pdf. [Consultado em: 23 de Junho de 2020].

Schläpfer, M., Bettencourt, L. M., Grauwin, S., Raschke, M., Claxton, R., Smoreda, Z., West, G., e Ratti, C. (2014). The scaling of human interactions with city size. Journal of the Royal Society Interface, 11(98), 20130789. 
Hey, T., Tansley, S., e Tolle, K. (2009) The

Fourth Paradigm: Data-Intensive Scientific

Discovery. Redmond, Microsoft Research. 